\title{
MONITORING OF HEAVY METALS BY ENERGY DISPERSIVE X-RAY FLUORESCENCE SPECTROMETRY*
}

\author{
Karl E. Lorber $\dagger$ \\ (Received 7 Sept 1985, revised 28 October 1985)
}

\begin{abstract}
Fundamentals and salient features of a projected heavy metals monitoring system are discussed. Determination of mass concentrations is achieved by using a new dust emission monitor, consisting of a "Zero Pressure Probe" for isokinetic sampling, which is connected to a "Longitudinally Oscillating Band" mass detector. The thin dust layer, precipitated on the surface of heat resistant filter tapes could be analysed nondestructively by energy-dispersive X-ray fluorescence (EDXRF). Using a small portable EDXRF-system with radioisotope-excitation, quasi-continuous monitoring of particulate heavy metals ( $\mathrm{Cd}, \mathrm{Hg}, \mathrm{Pb}, \mathrm{Cu}, \mathrm{Sb}, \mathrm{Zn}, \mathrm{Sn}$ ) suspended in incinerator stack gases is expected to be feasible. Another application of EDXRF-analysis is the reported investigation of electrostatic precipitator ash (ESP-fly ash). Using the "Linear Multi-Elemental-Standard Addition Method" as a fast and simple routine technique, results are reported for the concentrations of $\mathrm{Pb}, \mathrm{Cd}, \mathrm{Cu}$ and $\mathrm{Zn}$ in fly ash samples which had been collected in different fields (hoppers) of the electrostatic precipitator.
\end{abstract}

Key Words-Waste incineration, precipitator ash, EDXRF analysis, particulate heavy metals, stack gas analysis.

\section{Introduction}

Waste incinerators may be considered as variable emission sources which are prone to frequent fluctuations and rapid changes in stack gas concentrations. To meet the practical requirements of monitoring heavy metals emitted by the combustion of waste, a fast and reliable analytical method has to be applied in combination with a continuous or quasicontinuous stack gas sampling system. Out of various analytical methods taken into consideration, energy-dispersive X-ray fluorescence (EDXRF) spectrometry offers the advantages of non-destructive, simultaneous multi-element analysis with a high degree of automatization.

Recently, an R\&D project has been started at the Technical University in Berlin. dealing with the monitoring of particulate heavy metals suspended in incinerator stack gases. In this paper, principles and salient features of the projected Heavy Metals Monitoring System are discussed and a simple and fast method is reported for the determination of heavy metals in flyash which was collected in different fields of an electrostatic precipitator (ESP).

\section{Principles of a new heavy metals monitoring system}

For quasi-continuous monitoring of particulate heavy metals suspended in incinerator

\footnotetext{
*Presented at the ISWA Specialized Seminar Incinerator Emissions of Heary Molals and Particulates. Copenhagen, 18-19 September 1985.

†Department of Air Chemistry KF 3, Institute for Environmental Protection (ITU), Technical University Berlin, D-1000, Berlin 12, F.R.G.
} 
stack gases, the following approach is suggested, using an integrated system which contains a newly developed dust concentration measuring instrument (Gast \& Kramm 1984), on-line connected to a modified EDXRF spectrometer. The dust measuring instrument used in the $\mathrm{R} \& \mathrm{D}$ project had been developed at the Institut für Meß- und Regelungstechnik, Technical University Berlin. It consists of an isokinetic stackgas sampling device (Zero Pressure Probe) and a Longitudinally Oscillating Band mass detector.

\subsection{Isokinetic stackgas sampling with the "Zero-Pressure-Probe"}

The basic principles of the "Zero-Pressure-Probe" (Nulldrucksonde) are demonstrated in Fig. 1. The probe works with an isokinetic flow divider, based on measurements of the pressure difference between a sensor channel (a) and the main channel (b).

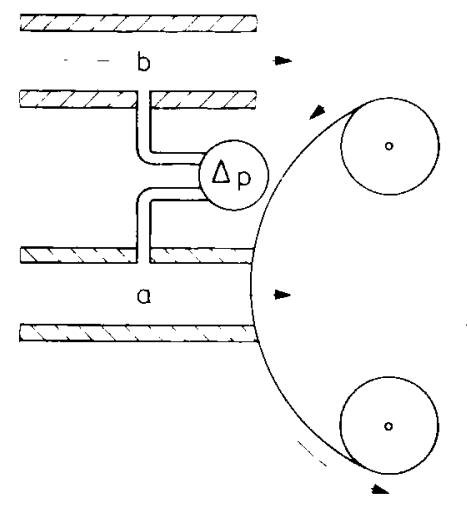

Fig. 1. Operating fundamentals of the Zero Pressure Probe for isokinetic stack gas sampling. a, Sensor channel; b, main channel.

Parallel to flow direction, sensor channel (a) and main channel (b) are placed in the stack gas stream. At the inside wall of the open main channel (b), the static pressure $P_{\text {stat }}$ occurs. Suppose that sensor channel (a) is closed on the right-hand side, the inside wall pressure will be the sum of the static pressure $P_{\text {stat }}$ and the dynamic pressure $P_{\text {dyn }}$. Thus, according to the Bernoulli equation, the pressure difference between (a) and (b) becomes: $\Delta P=\mathrm{P}_{\mathrm{d} y \mathrm{n}}$. If the sensor-channel (a) is closed with a permeable filter tape as in Fig. 1 , isokinetic sampling is achieved as soon as the pressure difference $\Delta P$ becomes zero. As the flow resistance increases with the thickness of the dust layer collected on the filter tape, the revolution $\left(\mathrm{r} \mathrm{m}^{-1}\right)$ of the pump is regulated accordingly by an electrical signal from the pressure difference transmitter. Thus, isokinetic sampling is continuously controlled by keeping the pressure difference $\Delta P$ at zero and any change in the stack gas velocity is immediately accounted for.

This automatically controlled Zero Pressure Probe system offers some crucial advantages compared to conventional stack gas sampling devices. Using a Pitot tube or Prandt-Staurohr arrangement like the standard VDI 2066 (VDI, 1979) provided by the Association of German Engineers, the requirements on trained manpower will be much higher and the accuracy of isokinetic sampling will be significantly lower. A schematic drawing of the integrated Zero Pressure Probe is shown in Fig. 2. 


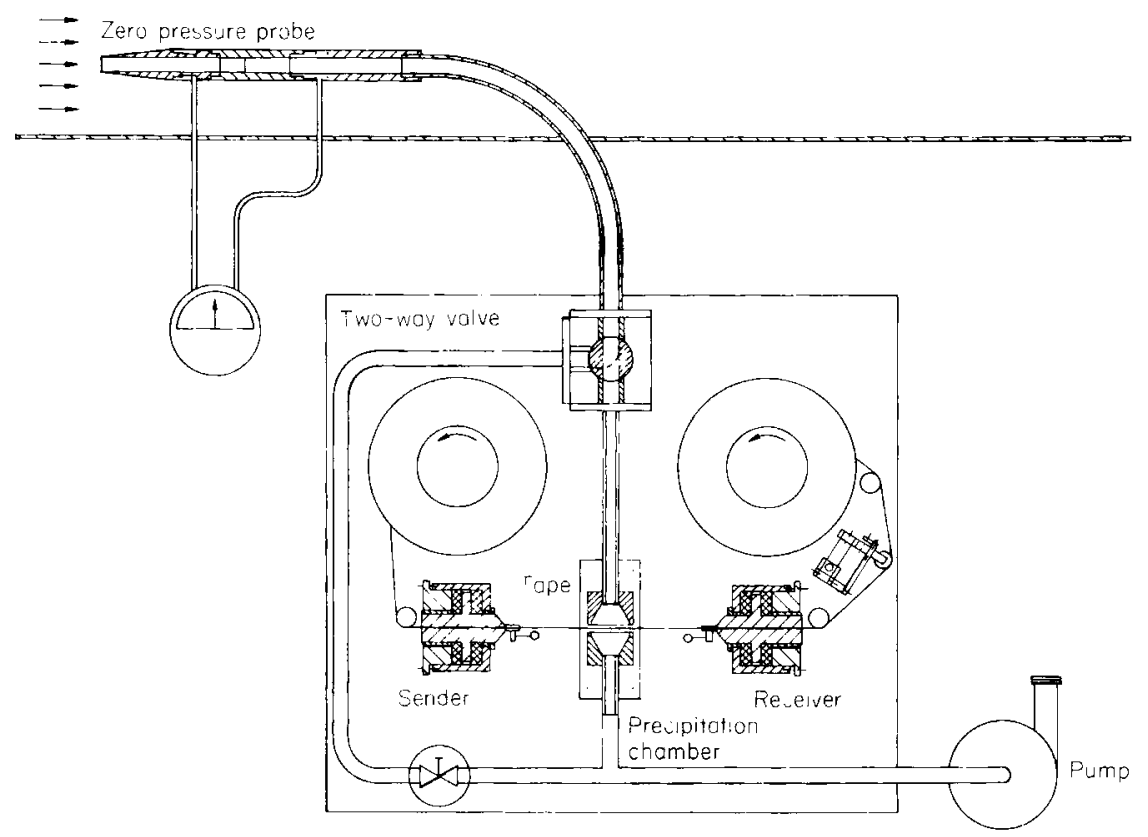

Fig. 2. Dust emission monitor with integrated Zero Pressure Probe and Longitudinally Oscillating Band mass detector. (Courtesy of Th. Gast and U. Kramm, Technical University, Berlin, F.R.G.).

\subsection{Dust concentration measurement by means of a longitudinally oscillating filter tape}

The fundamentals of the Longitudinally Oscillating Band mass detector are shown in Fig. 2. Using the integrated Zero Pressure Probe, a representative partial flow is sucked out of the stack gas. Via the two-way valve, the sampled gas is pumped to the precipitation chamber where the suspended particulate matter (dust) is mechanically deposited on the surface of a filter tape. Alternatively, electrostatic precipitation of the charged particles is possible too. In case of incinerator stack gas sampling, the gas temperature (about $250-230{ }^{\circ} \mathrm{C}$ in the stack) is cooled down to $140{ }^{\circ} \mathrm{C}$ and heat resistant Teflon (PTFE) type membrane filter tapes are used for dust collection. Mass detection of the precipitated dust is achieved quasi-continuously by measuring the change in the resonant frequency of the longitudinally oscillating filter tape.

According to the following equation (1), the piezo-electrically exited filter tape will change its resonant frequency when the precipitated dust mass participates in oscillation:

$$
M=m\left(\frac{f_{0}^{2}}{f_{\mathrm{m}}^{2}}-1\right),
$$

where $M=$ precipitated dust mass, $m=$ filter band mass (before precipitation), $f_{\mathrm{o}}=$ resonant frequency of filter band (before precipitation), $f_{\mathrm{m}}=$ decreased resonant frequency of filter band (after precipitation). The voltage applied to the piezo-electrical sender will cause longitudinal oscillation of the filter tape. As already mentioned, the change in the resonant frequency of the oscillating band is a function of the precipitated dust mass.

For detection, the piezo-electrical receiver transfers the force of oscillation into voltage again. Finally, the input voltage of the sender and the output voltage of the receiver are 
displayed on the oscillograph. In the automatic operation mode of the instrument, it is fully controlled by a HP 85 calculator which converts the output signal of the resonant frequency into the mass of the precipitated dust. This new quasi-continuously operating dust emission monitor has some essential advantages compared to conventional measuring instruments. As the output signal does not depend on the chemical composition or the physical properties of the dust particles collected, no empirical conversion factors are necessary for calculating the mass. This is not true for radiometric or optical dust monitors, where the measured extinction values have to be converted into mass units by using an empirical factor between gravimetric determination and the indirect method used. The correlation coefficient may vary between 0.47 and 0.83 (Müller 1983). This new dust emission monitor, which recently became commercially available, has obtained a proven reliability in the mean time when it was used in the field. For a glass fibre filter tape, the observed sensitivity is $35 \mathrm{~Hz} \mathrm{mg}^{-1}$. A minimum detection limit of $0.1 \mathrm{mg}$ or less seems to be possible by optimizing the system. The filter collection efficiency can be determined by the device used for testing high load sampling systems (Søby \& Mosbaek 1983).

\subsection{Photometric determination of the "average" particle size}

In collaboration between the Technical University Berlin and the Nuclear Research Centre Karlsruhe, a modified version of the reported dust emission monitor has been developed (Kernforschungszentrum Karlsruhe 1985). Using the mass concentration extinction size analyser (MESA) instrument, simultaneously the "average"-particle size of the dust can be determined by measuring the Lambert-Beer extinction factor,

$$
E=\ln \frac{I_{0}}{I}
$$

with the attached photometer.

From the equation

$$
d_{\mathrm{em}}=\frac{3 \times l \times C_{\mathrm{m}}}{\mathrm{E} \times \rho}
$$

where $I_{0}$ and $I$ are the intensities of the transmitted light before and after absorbance, respectively, the so-called "average" particle size of the suspended dust particles can be calculated, when the mass concentration, $C_{\mathrm{m}}$, and the extinction, $E$, is measured, and the path length of the transmitted light, $l$, and the density of the particulate matter, $\rho$, are known. The reported working-range of the photometer for $C_{\mathrm{m}}$ is between $10^{-3}$ and $1.0 \mathrm{gm}^{-3}$, and the particle size detection range is between 0.3 and $10.0 \mu \mathrm{m}$.

\subsection{Quantitative multi-elemental analysis by $X$-ray fluorescence spectrometry}

For the quasi-continuous monitoring of particulate heavy metals suspended in incinerator stack gases, it is suggested that an EDXRF system is connected on-line to the MESA instrument reported above. In Fig. 3, the non-destructive, simultaneous multielemental analysis of the fine dust layer collected on the filter tape is schematically shown.

X-Ray fluorescence (XRF) analysis of fine dust layers collected on the surface of membrane filters is advantageous, as long as the precipitated mass is in the range of 1-10 mg dust $\mathrm{cm}^{-2}$ filter surface (Lorber et al. 1978). In this case, the sample can be considered as a "thin film" which does not show any serious matrix effects. If the surface is smooth 


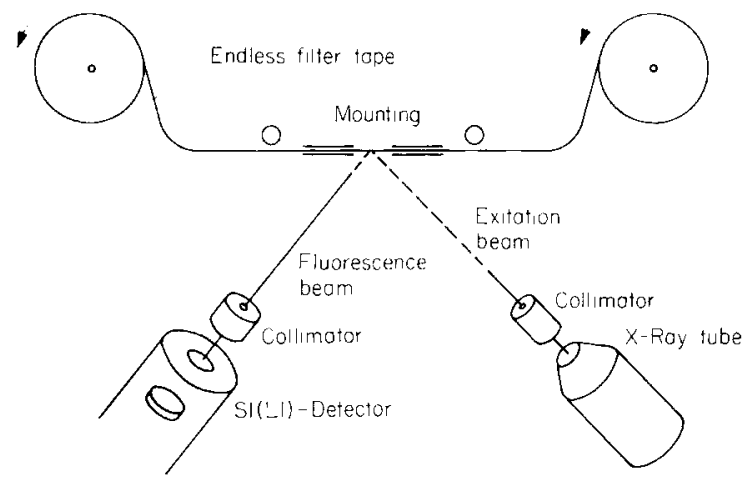

Fig. 3. Suggested approach for the quasi-continuous monitoring of heavy metals (R\&D project, Technical University, Berlin, F.R.G.): on-line analysis of the collected dust by means of energy dispersive. X-ray fluorescence.

enough, no particle effect will occur, and the measured fluorescence intensities of the elements will be a linear function of their concentrations. Thus, no sample preparation seems to be necessary when appropriate membrane filter tapes (e.g. "fluoropore") are used. (In our experience, glass fibre filter materials are not adequate because of the rough surface and the relatively high blank values due to contamination.)

Another problem to solve is the calibration of the analysis system. In principle, two different ways might be taken into consideration: the application of synthetic "thin-film" standards (Billiet et al. 1980) or the re-calibration of the XRF system by atomic absorption spectrometry (AAS) analysis of the non-destructively investigated filter tape samples. At present, a series of filters are analysed by AAS after they had been measured by the EDXRF system.

Early results indicate that the minimum detection limits (MDL) for heavy metals are in the range between $1-10 \mu \mathrm{g} \mathrm{cm}^{-2}$. Using the Totally Reflecting Sample Holders technique (Ketelsen \& Knöchel 1983) or by improving the geometry of the sample chamber, lower minimum detection limits and higher sensitivities are achievable.

In a first approach, a TRACOR NORTHERN TN 2000-EDXRF-system is being modified for the automatic analysis of filter tapes which are supplied by the dust emission monitor developed at the Institut für Meß- und Regelungstechnik in Berlin. For this purpose, the filter tape has to be fitted into a conventional sample chamber where online analysis is not possible.

The final goal of the R \& D project reported is the development of a quasi-continuous heavy metals monitor with on-line analysis of the endless filter tape. To meet these expectations, we have the intention to use a small portable EDXRF system with radioisotope ( $\gamma$-source) excitation and a simple detector system with a limited number of fixed channels. This type of analysis system is supposed to be fully compatible with the already existing dust emission monitor.

Based on the observed minimum detection limits between 1 and $10 \mu \mathrm{g} \mathrm{cm}^{-2}$, the necessary amounts $(\mathrm{mg})$ of dust, which have to be precipitated on the tape for EDXRF detection of different heavy metals are estimated in Table 1 . It can be seen from Table 1, that EDXRF monitoring of $\mathrm{Cd}, \mathrm{Hg}, \mathrm{Pb}, \mathrm{Cu}, \mathrm{Sb}, \mathrm{Zn}$ and $\mathrm{Sn}$ is expected to be feasible, due to sufficient concentrations of these elements in the emitted fine incinerator dust. For $\mathrm{Ni}, \mathrm{Cr}$, As and V, EDXRF detection is questionable and monitoring could be possibly done only after extended sampling times. Monitoring of $\mathrm{Tl}$ will not be possible at all. 


\section{TABLE 1}

Average concentrations of heavy metals in emitted fine dust from incinerators (Vogg 1984; Lorber 1984) and estimated necessary amounts of dust collected on the tape for energy dispersive X-ray fluorescence detection

\begin{tabular}{|c|c|c|c|}
\hline \multirow[b]{2}{*}{ Element } & \multirow{2}{*}{$\begin{array}{l}\text { Mass-fraction } \\
\text { in emitted fine } \\
\text { dust }\left(\mu \mathrm{g} \mathrm{g}^{-1}\right)\end{array}$} & \multicolumn{2}{|c|}{$\begin{array}{l}\text { Precipitated dust mass } \\
\text { necessary for } \\
\text { EDXRF-detection }(\mathrm{mg})\end{array}$} \\
\hline & & MDI*: $10 \mu \mathrm{g} \mathrm{cm}^{-2}$ & $\mathrm{MDI}^{*}: 1 \mu \mathrm{g} \mathrm{cm}^{-2}$ \\
\hline$\overline{\mathrm{Cd} \dagger}$ & $1500-2000$ & $7-5$ & $0.7-0.5$ \\
\hline $\mathrm{Hg} \dagger$ & $1000-2000$ & $10-5$ & $1-0.5$ \\
\hline $\mathrm{TI}+$ & 1 & 10,000 & 1000 \\
\hline $\mathrm{Nis}$ & 100 & 100 & 10 \\
\hline $\mathrm{Cr} \xi$ & $200-300$ & $50-30$ & $5-3$ \\
\hline As\$ & 100 & 100 & 10 \\
\hline $\mathrm{Pb} t$ & 30,000 & 0.3 & 0.03 \\
\hline $\mathrm{Cu}{ }^{\dagger}$ & 3000 & 3 & 0.3 \\
\hline $\mathrm{Sb}^{\dagger}$ & 3000 & 3 & 0.3 \\
\hline$V \S$ & 100 & 100 & 10 \\
\hline $\mathrm{Zn} \dagger$ & 80,000 & 0.1 & 0.01 \\
\hline Smt & 4000 & 2.5 & 0.25 \\
\hline
\end{tabular}

${ }^{*} \mathrm{MDL}=$ Minimum detection limit.

† Sufficient sensitivity for monitoring.

† Monitoring not possible.

$\S$ Monitoring questionable.

\section{Determination of heavy metals in ESP-collected fly ash}

Another application of EDXRF multi-elemental analysis is the determination of heavy metals in fly ash. The most common methods currently used for fly ash analysis are AAS or wet chemistry methods defined in ASTM-C311 (American Society for Testing and Materials 1981). However, the requirements in time and trained manpower are rather high compared to X-ray fluorescence spectrometry. For EDXRF analysis of heavy metals in fly ash, different techniques of sample preparations are commonly used, as follows.

(1) Borate fusion technique: by using $\mathrm{Li}_{2} \mathrm{~B}_{4} \mathrm{O}_{7}$ as a fluxing agent, the sample is converted into a borate-glass pellet. To avoid matrix-effects of the "non-infinitely-thick" samples, a heavy absorber (such as $\mathrm{MoO}_{3}$ ) is added to the flux. Calibration is achieved by a set of synthetic borate glass standards (Lorber et al. 1978).

(2) Pressed pellet technique: the finely ground fly ash is mixed with a briquetting agent (like cellulose-powder) and pressed to "infinitely thick" pellets. Quantification is preferentially done by the linear multi-elemental standard addition technique.

(3) Loose powder technique: A loose powder of finely ground fly ash (about $3 \mathrm{~g}$, passing through a $106 \mu \mathrm{m}$ sieve) is filled into a Mylar cup. Excitation of the "infinitely-thick" layer of powder takes place through a thin polycarbonate foil on the bottom of the cup. Quantification is accomplished by the linear multi-elemental standard addition technique. In case of matrix effects - which result in non-linear response curves-the powdered sample can be diluted with $\mathrm{SiO}_{2}$ to reduce concentrations.

A comparison of the three sample preparation methods mentioned above revealed that the most simple Loose Powder technique enables fast routine analyses of fly ash. As the agreement of the results obtained with different methods was satisfactory, this technique 
has been used for the following investigation of fly ash, precipitated in different sections (first and second field) of the ESP.

\subsection{Linear multi-elemental standard addition method}

Using an agate mortar or a tungsten carbide mill, the original fly ash sample is ground to a fine powder which is divided into four equal portions. One portion remains as it is, but the other portions are "doped" with known amounts of a multi-elemental standard which can be added in powder form. After carefully grinding and mixing, the four partial samples are filled into Mylar-cups and measured by EDXRF.

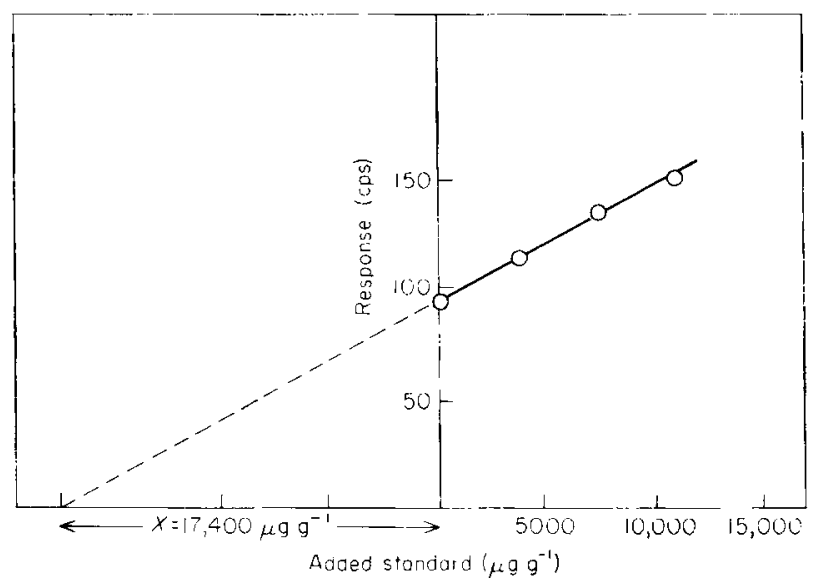

Fig. 4. Linear multi-elemental Standard Addition Technique: determination of lead in the fly ash sample $\mathbf{R}$ 1.4. (Ash collected in the hopper of the second field of electrostatic precipitator investigated. ---, Extrapolation of line. Correlation coefficient, $r^{2}=0.9983$.

As shown in Fig. 4, linear extrapolation is possible, as long as the following criteria are met:

(1) The powdered sample must be homogeneous in respect of composition and particle size.

(2) For the excitation- and fluorescence radiation, the analysed layer of loose powder must be "infinitely thick".

(3) The bulk density of the four partial samples should be the same.

(4) The concentrations of the elements investigated and standard added should not be to high (less than about $2-5 \%$ ), or powder dilution techniques should be applied.

\subsection{Results}

Using the same scale for display, the EDXRF-spectra of three different fly ash samples are shown in Figs 5-6 (Cd, which has been analysed at higher tube voltage, is not visible on this display). A comparison of the EDXRF spectra reveals the well known fact, that the concentration of heavy metals in incinerator fly ash is significantly higher than in fiy ash from coal fired power stations.

When an ESP is used with two electrical fields in flow direction, the major portion of 


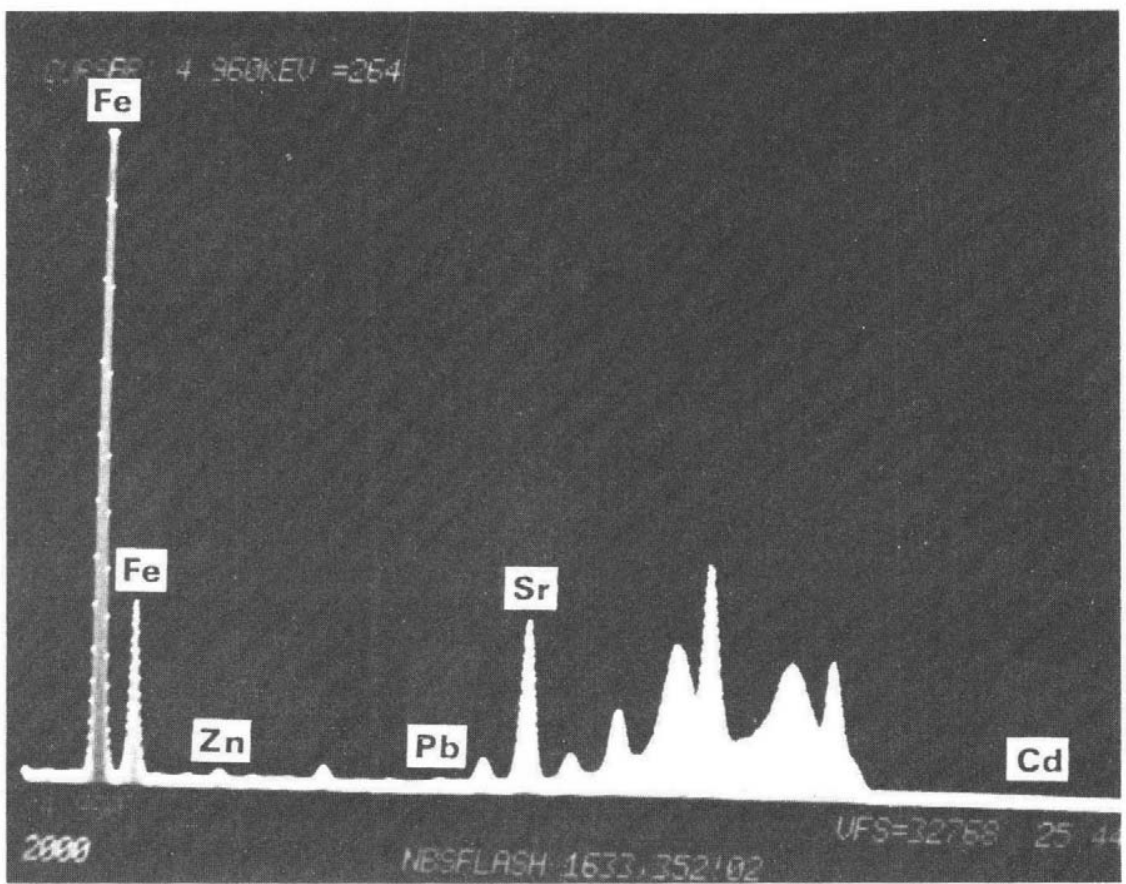

Fig. 5. Energy dispersive X-ray fluorescence spectrum of NBS fly ash SRM 1633 from coal fired power stations.

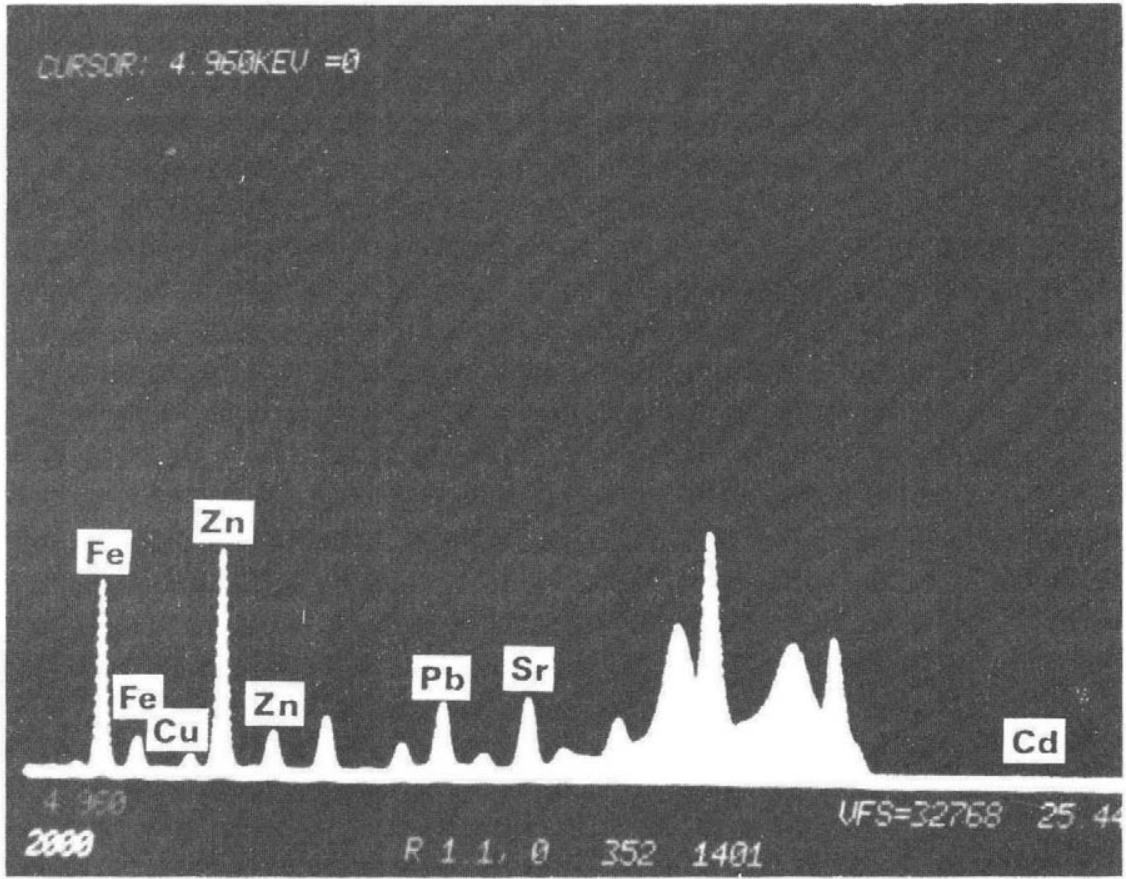

Fig. 6. Energy dispersive X-ray fluorescence spectrum of incinerator fly ash from Berlin (collected from the hopper of the first field of the investigated electrostatic precipitator). 


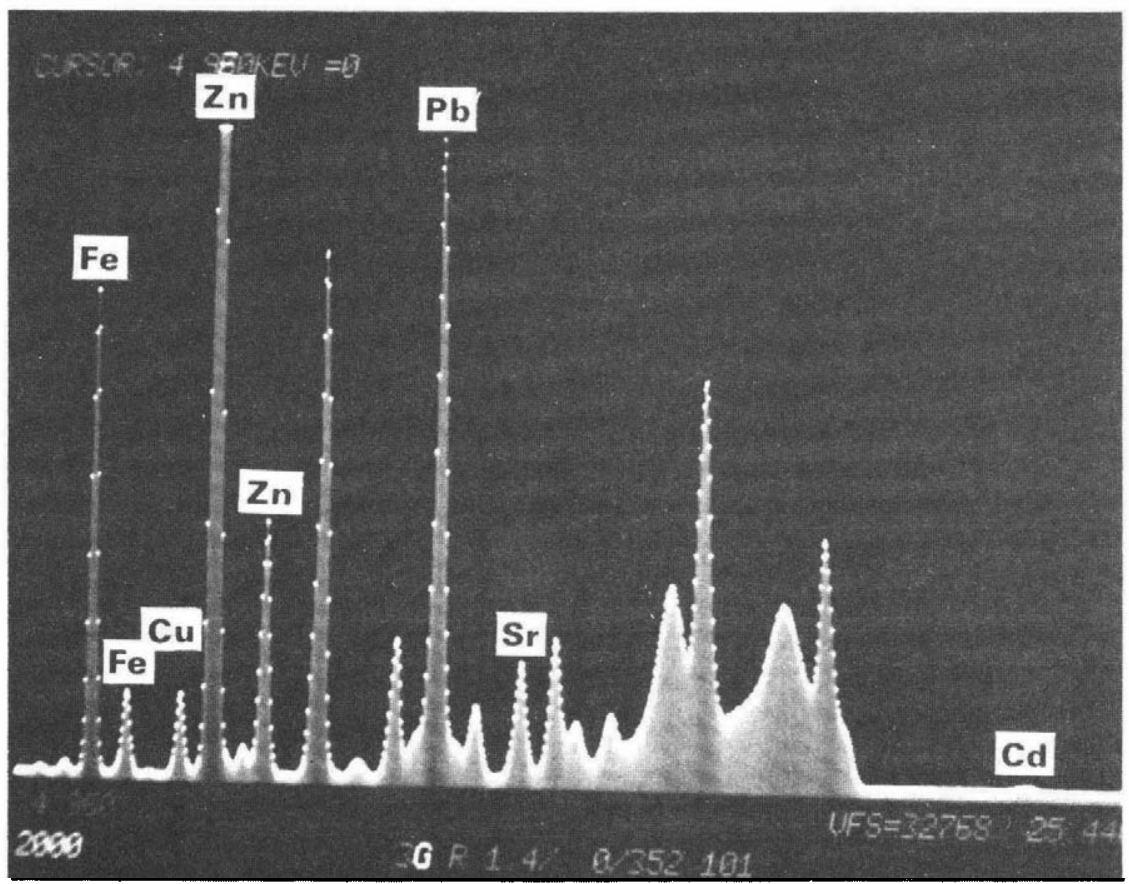

Fig. 7. Energy dispersive X-ray fluorescence spectrum of incinerator fly ash from Berlin (collected from the hopper of the second field of the investigated electrostatic precipitator.

fly ash is precipitated in the first field and only a rather small portion is collected in the hoppers of the second field. But due to the well known prevalence of heavy metals on the surface of small particles (Lorber 1980), the concentration of these elements ( $\mathrm{Pb}, \mathrm{Cd}$, $\mathrm{Cu}, \mathrm{Zn}$, etc.) is significantly higher in the fly ash sample collected in the hopper of ESP field 2.

The results discussed above are quantitatively presented in Table 2 .

TABLE 2

Quant tative results for different fly ash samples, using the energy dispersive X-ray fluorescence detection Linear Multi-Elemental-Standard Addition technique. The NBS-SRM 1633* was used for verification. (Confidence limit $=95 \%$, number of determinations $=7$ )

\begin{tabular}{|c|c|c|c|c|}
\hline \multirow[b]{3}{*}{ Element } & \multicolumn{4}{|c|}{ Mass fraction in fly ash $\left(\mu \mathrm{g} \mathrm{g}^{-1}\right)$} \\
\hline & \multicolumn{2}{|c|}{ NBS-SRM 1633} & \multirow{2}{*}{$\begin{array}{l}\text { Pre-Filter Ash } \\
\text { (ESP field 1)* }\end{array}$} & \multirow{2}{*}{$\begin{array}{l}\text { Post-Filter Ash } \\
\text { (ESP field 2) }\end{array}$} \\
\hline & Certified & Found & & \\
\hline$\overline{\mathrm{Cd}}$ & $1.45 \pm 0.06$ & - & $168 \pm 12$ & $654 \pm 31$ \\
\hline $\mathrm{Pb}$ & $70 \pm 4$ & $81 \pm 12$ & $2480 \pm 89$ & $18850 \pm 662$ \\
\hline $\mathrm{Cu}$ & $128 \pm 5$ & $125 \pm 15$ & $500 \pm 47$ & $2570 \pm 253$ \\
\hline $\mathrm{Zn}$ & $210 \pm 20$ & $223 \pm 27$ & $5830 \pm 492$ & $23970 \pm 1181$ \\
\hline
\end{tabular}

* Standard reference material, from U.S. National Bureau of Standards. 


\section{Conclusions}

Energy dispersive X-ray fluorescence spectrometry offers the advantages of fast and simple multi-elemental analyses, which are needed for monitoring of heavy metals mobilized by incineration of waste. Using a newly developed dust emission monitor system, which contains a Zero Pressure Probe connected to a Longitudinally Oscillating Band mass detector, fine dust from stack gas can be collected on a filter tape. The results of EDXRF analysis of filter tapes indicate that for heavy metals the minimum detection limits are in the range of $1-10 \mu \mathrm{g} \mathrm{cm}^{-2}$. Based on known stack gas concentrations, the minimum amounts of fine dust required for stack gas monitoring of heavy metals are estimated. It is expected, that for $\mathrm{Cd}, \mathrm{Hg}, \mathrm{Pb}, \mathrm{Cu}, \mathrm{Sb}, \mathrm{Zn}$ and $\mathrm{Sn}$ the sensitivity of the EDXRF analysis system, connected on-line to the dust emission monitor, is sufficient.

Using the simple Loose Powder Standard Addition technique, fly ash can be quickly analysed for heavy metals. When filter ash from different fields of the ESP was investigated, it was shown that the concentrations of $\mathrm{Cd}, \mathrm{Pb}, \mathrm{Cu}$ and $\mathrm{Zn}$ are significantly higher in the post-filter ash of ESP-field 2 compared to the pre-filter ash of ESPfield 1 .

\section{References}

American Society for Testing and Materials (1981), Annual Book of ASTM-Standards, Part 14. ASTM Philadelphia, U.S.A.

Billiet, J., Dams, R. \& Hoste, J. (1980), Multielement thin film standards for XRF analysis, $X$-Ray Spectrometry, 9(4).

Gast, Th. \& Kramm, U. (1984), Abschlußbericht zum Forschungsvorhaben Kraftwerksemissionsmessungen. Bundesministerium für Forschung und Technologie (BMFT) (Final report: Measurement of emissions from power stations. Ministry of Research \& Technology) Reference No. I-ET 1141 A. Ministry of Research and Technology, F.R.G.

Kernforschungszentrum Karlsruhe (1985), Koordinationsstelle Technologietransfer (Nuclear research centre Karlsruhe, coordination bureau for technology transfer), personal communications.

Ketelsen, P. \& Knöchel A. (1983), Multielementanalyse von Aerosolen mit Hilfe der Röntgenfluoreszenzanalyse mit totalreflektierendem Probenträger (TRFA). (Multi-element analysis of aerosols by XRF with totally reflecting sample holder). Publication of the Institut für Anorganische und Angewandte Chemie, Universität Hamburg, F.R.G.

Lorber, K. E., Wegscheider, W., Spitzy, H., Heinrich, K. F. J. \& Pella, P. A. (1978), Development of EDXRF-Techniques for the Quantitative Multielement Analysis of Environmental Samples, Mikrochim. Acta, $I$.

Lorber, K. E. (1980), Müllverbrennung und Schwermetallemission (Waste incineration and emission of heavy metals), Müll und Abfall, 6 .

Lorber, K. E. (1985), Incineration of RDF and incineration of total waste-comparison of emissions. In Sorting of Household Waste and Thermal Treatment of Waste, Commission of the European Communities (Ferranti \& Ferrero, Eds). Elsevier Applied Science Publishers, London and New York.

Müller, J. (1983), Test of sampling instruments for suspended particulate matter in ambient air. Umweltbundesamt, Frankfurt/Main. Contribution to the poster-session during the Seminar: Aerosols in Science, Medicine and Technology, 14-16 September 1983, München, F.R.G.

Søby F. \& Mosbaek, H. (1983), Assessment of stack gas emitted trace metals from incineration: determination of filter collection efficiency by condensational enlargement of penetrating submicron particles, Waste Management \& Research 1, 255-266.

VDI (1979) Verein Deutscher Ingenieure: VDI 2066. Messen von Partikeln. Staubmessungen in strömenden Gasen. Gravimetrische Bestimmung der Staubbeladung (Assoziation of German Engineers, VDI-guidelines: Measurement of particles. Measurement of dust in moving gases. Gravimetrical determination of the dust load). VDI-Kommission Reinhaltung der Luft, VDIVerlag GmbH, Düsseldorf, F.R.G. 
Vogg, H. (1984), Verhalten von (Schwer)-Metallen bei der Verbrennung kommunaler Abfälle (Behavior of heavy metals during incineration of municipal refuse), Chem-Ing.-Techn., 56. 